\title{
Transcriptional Profile of Aedes aegypti Leucine-Rich Repeat Proteins in Response to Zika and Chikungunya Viruses
}

\author{
Liming Zhao *(1), Barry W. Alto and Dongyoung Shin \\ Florida Medical Entomology Laboratory, University of Florida, 200 9th Street South East, \\ Vero Beach, FL 32962, USA; bwalto@ufl.edu (B.W.A.); dshin@ufl.edu (D.S.) \\ * Correspondence: lmzhao@ufl.edu; Tel.: +1-772-778-7200
}

Received: 30 December 2018; Accepted: 28 January 2019; Published: 31 January 2019

check for updates

\begin{abstract}
Aedes aegypti (L.) is the primary vector of chikungunya, dengue, yellow fever, and Zika viruses. The leucine-rich repeats (LRR)-containing domain is evolutionarily conserved in many proteins associated with innate immunity in invertebrates and vertebrates, as well as plants. We focused on the AaeLRIM1 and AaeAPL1 gene expressions in response to Zika virus (ZIKV) and chikungunya virus (CHIKV) infection using a time course study, as well as the developmental expressions in the eggs, larvae, pupae, and adults. RNA-seq analysis data provided 60 leucine-rich repeat related transcriptions in Ae. aegypti in response to Zika virus (Accession number: GSE118858, accessed on: August 22, 2018, GEO DataSets). RNA-seq analysis data showed that AaeLRIM1 (AAEL012086-RA) and AaeAPL1 (AAEL009520-RA) were significantly upregulated 2.5 and 3-fold during infection by ZIKV 7-days post infection (dpi) of an Ae. aegypti Key West strain compared to an Orlando strain. The qPCR data showed that LRR-containing proteins related genes, AaeLRIM1 and AaeAPL1, and five paralogues were expressed 100-fold lower than other nuclear genes, such as defensin, during all developmental stages examined. Together, these data provide insights into the transcription profiles of LRR proteins of Ae. aegypti during its development and in response to infection with emergent arboviruses.
\end{abstract}

Keywords: Aedes aegypti; Leucine-Rich Repeat Proteins; Zika virus; chikungunya virus; immune responses; gene expression

\section{Introduction}

The leucine-rich repeats (LRR)-containing domain is noted to be evolutionarily conserved among many proteins correlated with innate immunity in an array of organisms, including invertebrates, vertebrates, and plants [1]. Innate immunity is a conserved host response that serves as the host's first line of defense by sensing pathogen-associated molecular patterns through germline-encoded pattern recognition receptors [2]. For example, in mammals, activation of antimicrobial defenses and stimulation of the adaptive immune response is mediated by Toll-like receptors (TLRs) and nucleotide-binding oligomerization domain (NOD)-like receptors through the LRR domain in response to pathogens [3].

Mosquitoes lack an adaptive immune system [4] and so they are fully dependent on mounting an innate immune response to fight infection [5-8]. Research indicates that mosquito vectors and mosquito cell lines produce both humoral and cellular immune responses against invading pathogens $[9,10]$. Leucine-rich repeat immune proteins (LRIMs) are a mosquito-specific family of putative innate receptors [11-13]. Two LRR proteins, LRIM1 (leucine-rich repeat immune protein 1) and APL1 (Anopheles Plasmodium-responsive leucine-rich repeat 1), have been recognized as major mosquito 
factors that regulate parasite infection and parasite loads [14]. In Anopheles mosquitoes, LRIM1, APL1C, and LRR-containing proteins activate complement-like defense responses against malaria by forming a disulphide-bridge that interacts with thioester-containing protein 1 (TEP1), a complement C3-like protein [15,16]. APL1 of Anopheles gambiae is a family of variable LRR proteins required for cell-mediated protection as shown using a rodent model malaria parasite, Plasmodium berghei [17]. The An. gambiae APL1 genomic locus circumscribes three distinct genes (APL1A, APL1B, and APL1C), however, only the product of APL1C acts as a P. berghei antagonist [17]. LRIM1 and APL1C both play a significant role in the anti-Plasmodium response. Accordingly, silencing of these genes results in an altered response against Plasmodium infection [13]. A genome-wide study found that variations or polymorphisms in the LRIM1 and APL1C proteins were correlated with resistance and susceptibility to Plasmodium infection [18]. Additional studies offered mechanisms for controlling Plasmodium early during the infection process by targeting the ookinete or oocyst stages of oocyst $[19,20]$. Nonetheless, no detailed information has been generated for pathogen response by the LRR-containing proteins of immune factors in Aedes mosquitoes, responsible for transmitting multiple emerging arboviruses.

Aedes aegypti (L.) is a vector for transmitting emergent arboviruses, including chikungunya, dengue, yellow fever, and Zika viruses. Chikungunya fever is primarily transmitted to humans through mosquito vectors, Ae. aegypti and Ae. albopictus. It is a viral disease belonging to the family, Togaviridae, and genus, Alphavirus. Recent outbreaks of chikungunya fever occurred from 2004 to 2006 spanning Kenya in 2004 (Eastern/Central/Southern African, ECSA, CHIKV lineage) and the island of La Réunion in 2005-2006 (Indian Ocean CHIKV lineage). It later emerged in the New World in 2013 on St. Martin Island (Asian CHIKV lineage), eventually spreading throughout the Americas [21-24]. Over the span of the past 12 years, it is estimated that more than four million human cases of chikungunya infection have occurred worldwide [25]. Symptoms of infection include a rash, fever, headache, joint pain, and muscle pain [26] along with the chance of developing chronic musculoskeletal diseases [27]. There is currently no vaccine available for CHIKV. Accordingly, controlling the mosquito vectors is the primary method utilized to reduce the risk of disease transmission.

Zika virus (ZIKV) was first discovered in 1947 and belongs to the family, Flaviviridae, genus, Flavivirus (CDC 2016) [28]. Spreading to the Oceania region, ZIKV caused outbreaks on Yap Island in Micronesia and French Polynesia in 2007 and 2013, respectively. In 2015, ZIKV was found to have reached Brazil, spreading throughout the Americas [28,29]. It is estimated that 1.5 million people have been infected by ZIKV in Brazil [30]. ZIKV continues to spread to new areas. Transmission in the U.S. is a major public health risk, notably for the Gulf states, such as Florida and Texas, where ecological conditions are favorable for the primary vector, Ae. aegypti, as well as an increasing likelihood for virus introduction by imported cases. Manifestations of ZIKV take on different forms, the most serious of which include birth defects in humans [31] along with neurological complications that may result in Guillain-Barré syndrome, both of which are significant public health threats [32].

Mosquitoes respond to infection using an array of molecular signaling pathways and immune effector proteins. A focus on the immune system response of Ae. aegypti has unveiled a transcriptome analysis of genome-wide mechanisms that are implicated in defense against arbovirus infections [33-37]. No sequence-structure-function relationships of mosquito leucine-rich repeat immune proteins in $A e$. aegypti in response to arboviruses are available, though the LRR-proteins have been compared with An. gambiae and Culex quinquefasciatus [16]. Many studies of LRIM1 and APL1C have been reported as playing an important role in defense against Plasmodium in Anopheles [13-15]. However, there is no study of LRIM1 and APL1C in the Aedes in response to arboviruses. Therefore, we re-examined previous RNAseq data analysis and obtained 60 leucine-rich repeat related transcriptions of Ae. aegypti (Accession number: GSE118858, accessed on: August 22, 2018, https:/ / www.ncbi.nlm.nih.gov/gds / ?term=GSE118858) [34]. We examined the AaeLRIM1 and AaeAPL1 gene expressions in response to both ZIKV and CHIKV infection using a time course study. Additionally, we investigated the developmental expressions of these genes in the eggs, larvae, pupae, and adults. The current study aims to improve 
our understanding of the transcription profiles of Ae. aegypti LRR proteins during development and in response to arbovirus infection.

\section{Results}

2.1. Leucine-Rich Repeat Proteins Changes in Transcriptome of the Ae. aegypti Female Adult in Response to ZIKV Infection

To better our understanding of molecular interactions and the immune response to arbovirus of Ae. aegypti from Florida, we re-examined RNA-seq data to explore the changes in leucine-rich repeat proteins in the Ae. aegypti (Key West and Orlando strains) transcriptome in response to oral ingestion of ZIKV infected blood and ZIKV infection (Accession number: GSE118858, https:/ / www.ncbi.nlm.nih.gov/gds/?term=GSE11). Data were provided by RNA-seq analysis that generated 60 leucine-rich repeat related transcriptions in the Ae. aegypti genome in response to Zika virus (Table S1A-D and Table 1A-D). Specifically, female Ae. aegypti transcriptomic RNA-seq data showed that 23 genes related to leucine-rich repeat proteins (LRRP) were significantly upregulated during infection by ZIKA in 7-days post infection (dpi) Ae. aegypti Key West strains compared with Orlando strains. Additionally, 17 of these genes between the two strains were upregulated more than 2-fold (p-adj $\leq 0.01$; log2 fold change $> \pm 2.0$ ) in response to ZIKV 7 dpi (Table 1A). AaeLRIM1 (AAEL012086-RA) and AaeAPL1 (AAEL009520-RA) were significantly upregulated 2.5 and 3-fold (Table 1A). When comparing transcriptome profiles of two Ae. aegypti strains in response to the control (blood-feeding only), only three genes related to leucine-rich repeat proteins were significantly upregulated/downregulated in 7-days post infection Ae. aegypti in Key West strains compared with Orlando strains (Table 1B). AaeLRIM1 (AAEL010286-RA) was significantly upregulated 3-fold (Table 1B). Comparing ZIKV infected Key West Ae. aegypti with the Key West control at 7 dpi, four differentially expressed (DE) transcripts related to LRRP were significantly dysregulated (two upregulated and two downregulated, Table 1C). AaeLRIM1 (AAEL010286-RA, p-adj $5.0 \times 10^{-9}, \log 2$ fold change -3.3918 ) was significantly down-regulated (Table 1C). Analysis and comparison of mRNA expression profiles of Ae. aegypti Orlando strains following ZIKV infection indicated five LRRP related genes, including AaeAPL1 (AAEL009520-RA, p-adj $2.8 \times 10^{-4}, \log 2$ fold change -2.2074), were significantly dysregulated (downregulated) 7-days post infection (Table 1D). 
Table 1. (A) Female Aedes aegypti transcriptomic RNA-seq data show leucine-rich repeat proteins related genes significantly upregulated in the Zika infection in the Key West strain compared with the Orlando strain Aedes aegypti 7-days post infection (Log2FC, Log2 fold change); (B) female Aedes aegypti transcriptomic RNA-seq data show leucine-rich repeat proteins related genes significantly upregulated/downregulated in the control (uninfected blood-feeding only) in the Key West strain compared with the Orlando strain Aedes aegypti 7-days post injection; (C) female Aedes aegypti transcriptomic RNA-seq data show leucine-rich repeat proteins related genes significantly dysregulated in the Key West strain Aedes aegypti 7-days post infection with ZIKV compared with control (uninfected blood-feeding only) in the Key West strain; (D) female Aedes aegypti transcriptomic RNA-seq data show leucine-rich repeat proteins related genes significantly dysregulated in the Orlando strain Aedes aegypti 7-days post infection with ZIKV compared with control (uninfected blood-feeding only) in the Orlando strain.

\begin{tabular}{|c|c|c|c|}
\hline Transcript ID & $\log 2 \mathrm{FC}$ & p-adj & Gene Description \\
\hline \multicolumn{4}{|c|}{ (A) } \\
\hline AAEL001401-RA & 3.2722 & $2.3 \times 10^{-27}$ & leucine-rich immune protein (Short) \\
\hline AAEL001402-RA & 3.2947 & $3.3 \times 10^{-33}$ & leucine-rich immune protein (Short) \\
\hline AAEL001414-RA & 3.3721 & $2.1 \times 10^{-36}$ & leucine-rich immune protein (Short) \\
\hline AAEL001417-RA & 3.9484 & $2.1 \times 10^{-5}$ & leucine-rich immune protein (Short) \\
\hline AAEL001420-RA & 3.4335 & $1.6 \times 10^{-73}$ & leucine-rich immune protein (Short) \\
\hline AAEL001649-RA & -0.2792 & $4.6 \times 10^{-3}$ & leucine aminopeptidase \\
\hline AAEL002295-RA & 2.4545 & $1.9 \times 10^{-44}$ & leucine-rich transmembrane protein \\
\hline AAEL002615-RA & 2.2527 & $4.9 \times 10^{-12}$ & leucine-rich transmembrane protein \\
\hline AAEL003262-RA & 1.8141 & $3.9 \times 10^{-5}$ & leucine-rich transmembrane protein \\
\hline AAEL003408-RA & 1.3747 & $5.3 \times 10^{-6}$ & leucine-rich transmembrane protein \\
\hline AAEL003713-RA & 1.397 & $7.2 \times 10^{-4}$ & leucine-rich transmembrane protein \\
\hline AAEL003720-RA & 1.1688 & $5.6 \times 10^{-5}$ & leucine-rich transmembrane protein \\
\hline AAEL005762-RA & 2.2277 & $2.7 \times 10^{-5}$ & leucine-rich transmembrane protein \\
\hline AAEL006975-RA & 1.7989 & $8.7 \times 10^{-5}$ & leucine aminopeptidase \\
\hline AAEL007103-RA & 3.2536 & $7.5 \times 10^{-11}$ & leucine-rich immune protein (TM) \\
\hline AAEL009520-RA ${ }^{1}$ & 3.0383 & $4.5 \times 10^{-25}$ & leucine-rich immune protein (Long) \\
\hline AAEL010125-RA & 3.2598 & $7.0 \times 10^{-8}$ & leucine-rich immune protein (Coil-less) \\
\hline AAEL010128-RA & 4.8149 & $2.4 \times 10^{-7}$ & leucine-rich immune protein (Long) \\
\hline AAEL010656-RA & 2.7658 & $3.8 \times 10^{-7}$ & leucine-rich immune protein (Short) \\
\hline AAEL012086-RA & 2.4679 & $1.7 \times 10^{-12}$ & leucine-rich immune protein (Long) \\
\hline AAEL012092-RA & 2.1252 & $2.6 \times 10^{-28}$ & leucine-rich repeat protein \\
\hline AAEL012093-RA & 2.2739 & $1.9 \times 10^{-10}$ & leucine-rich transmembrane protein \\
\hline AAEL012255-RA & 4.2146 & $1.9 \times 10^{-4}$ & leucine-rich immune protein (Short) \\
\hline \multicolumn{4}{|c|}{ (B) } \\
\hline AAEL000243-RA & 5.2443 & $1.8 \times 10^{-26}$ & leucine-rich transmembrane protein \\
\hline AAEL009894-RA & -0.9167 & $2.9 \times 10^{-4}$ & leucine-rich immune protein (Coil-less) \\
\hline AAEL010286-RA & 3.0287 & $1.5 \times 10^{-5}$ & leucine-rich transmembrane protein \\
\hline \multicolumn{4}{|c|}{ (C) } \\
\hline AAEL000243-RA & -6.6469 & $7.6 \times 10^{-44}$ & leucine-rich transmembrane protein \\
\hline AAEL003408-RA & 2.1477 & $1.8 \times 10^{-4}$ & leucine-rich transmembrane protein \\
\hline AAEL009894-RA & 0.8665 & $9.7 \times 10^{-8}$ & leucine-rich immune protein (Coil-less) \\
\hline AAEL010286-RA & -3.3918 & $5.0 \times 10^{-9}$ & leucine-rich transmembrane protein \\
\hline \multicolumn{4}{|c|}{ (D) } \\
\hline AAEL009520-RA ${ }^{2}$ & -2.2074 & $2.8 \times 10^{-4}$ & leucine-rich immune protein (Long) \\
\hline AAEL010128-RA & -5.3692 & $2.8 \times 10^{-6}$ & leucine-rich immune protein (Long) \\
\hline AAEL001402-RA & -3.3551 & $6.1 \times 10^{-8}$ & leucine-rich immune protein (Short) \\
\hline AAEL010656-RA & -2.5623 & $4.4 \times 10^{-3}$ & leucine-rich immune protein (Short) \\
\hline AAEL012255-RA & -4.1624 & $3.1 \times 10^{-5}$ & leucine-rich immune protein (Short) \\
\hline
\end{tabular}

${ }^{1}$ AAEL009520-RA is the same gene as AAEL024406. ${ }^{2}$ AAEL009520-RA is the same gene as AAEL024406.

\subsection{Leucine-Rich Repeat Proteins AaeAPL1 Paralogues of Aedes aegypti}

Evolutionary analysis of eight paralogues of AeaAPL1 of the Ae. Aegypti were conducted in MEGA7 [38] (Figure S1). The data showed that AaeAPL1 was closely related to AaeLRIM4. The DNA sequences producing significant alignments between AaeAPL1 and AaeLRIM4 showed 44\% identity. 


\subsection{Developmental Regulation of AaeLRIM1 and AaeAPL1}

To understand how AaeLRIM1 and AaeAPL1 are regulated during the development of Ae. aegypti, qPCR was performed to examine the relative transcription levels of AaeLRIM1 and AaeAPL1 in eggs, larvae, pupae, and male and female adults (Figure 1A,B).
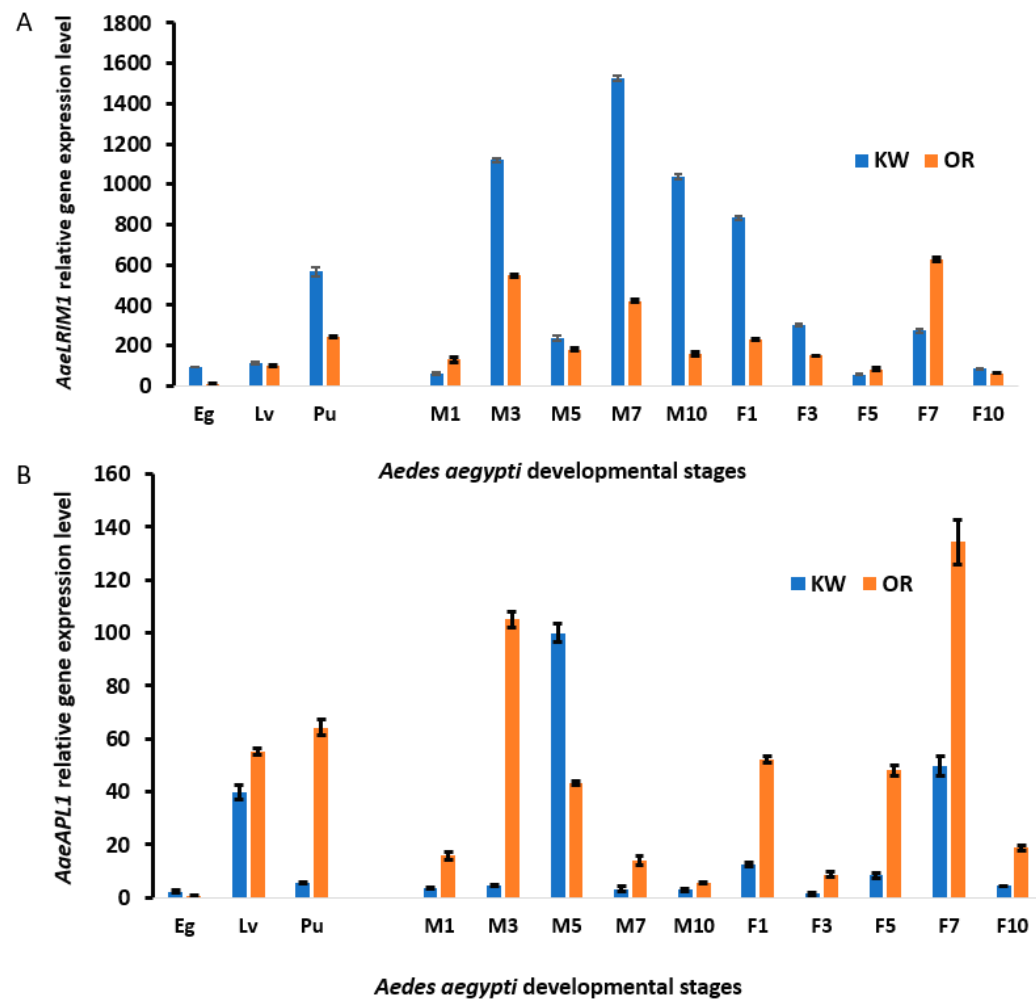

Figure 1. AaeLRIM1 and AaeAPL1 relative expression in the all developmental stages, from eggs (Eg), larvae (Lv), pupae (Pu), and adults Ae. aegypti, including male (M1, male 1-d-old; M3, male 3-d-old; M5, male 5-d-old; M7, male 7-d-old; and M10, male 10-d-old) and female (F1, female 1-d-old; F3, female 3-d-old; F5, female 5-d-old; F7, female 7-d-old; and F10, female 10-d-old) in the Key West strain (KW) and Orlando (OR) strain of Aedes aegypti. (A) AaeLRIM1; (B) AaeAPL1.

In addition, we also examined five paralogues of AeaAPL1, i.e., AaeLRIM3, AaeLRIM4, AaeLRIM15, AaeLRIM16, and AaeLRIM17, during developmental stages using qPCR (Figure S2A-E).

\subsubsection{AaeLRIM1 and AaeAPL1 RNA Profile in Immature Stages of Ae. aegypti}

Multivariate analysis of variance (MANOVA) showed significant effects of mosquito strain, developmental stage, and interaction of these factors (Table 2A). For the mosquito strain effect, Key West (permethrin resistant) had higher AaeLRIM1 than Orlando (permethrin susceptible strain) Ae. aegypti [34] (Figure 2). Standardized canonical coefficients showed that AaeLRIM1 and AaeAPL1 contributed similarly, but in opposite directions (Table 2A). In contrast, Orlando had higher AaeAPL1 than Key West Ae. aegypti (Figure 1). Standardized canonical coefficients showed that AaeLRIM1 contributed twice as much as AaeAPL1 for the significant developmental stage effect (Figure 2). For the developmental stage effect, LRIM1 was significantly different between developmental stages with increases associated between each stage (Figure 1). Gene expression of AaeAPL1 was significantly higher for the larval and pupal stages compared to the egg stage. However, AaeAPL1 was lower among pupae than larvae (Figure 1). For the significant interaction, we compared less than all possible treatment groups by which developmental stage was compared within a given strain (e.g., Key West eggs vs. Key West larvae). Standardized canonical coefficients showed a similar contribution of AaeLRIM1 and AaeAPL1 to the significant interaction, in opposite directions (Table 2A). Gene 
expression of AaeLRIM1 was higher for Key West than Orlando Ae. aegypti and occurred over a greater range. We observed significant increases in gene expression of AaeLRIM1 for each developmental stage for both Key West and Orlando Ae. aegypti (Figure 2). In contrast, gene expression of AaeAPL1 was higher for Orlando than Key West Ae. aegypti and occurred over a greater range. Gene expression of AaeAPL1 significantly increased for each developmental stage for both Key West and Orlando Ae. aegypti (Figure 2).

Table 2. (A) MANOVA results for strain and stage effects of the AaeLRIM1 and AaeAPL1 RNA profile in immature stages of Ae. aegypti. (B) MANOVA results for strain, sex, and age effects of the AaeLRIM1 and AaeAPL1 RNA profile in adult stages of Ae. aegypti.

\begin{tabular}{|c|c|c|c|c|c|}
\hline \multirow{2}{*}{ Treatment } & \multirow{2}{*}{ Pillai's Trace } & \multirow{2}{*}{ df (Numerator and Denominator) } & \multirow{2}{*}{$p$-value } & \multicolumn{2}{|c|}{ Standardized Canonical Coefficients } \\
\hline & & & & AaeLR1M1 & AaeAPL1 \\
\hline \multicolumn{6}{|c|}{ (A) } \\
\hline Strain & 0.99 & 2,11 & $<0.0001$ & 12.12 & -10.76 \\
\hline Stage & 1.99 & 4,24 & $<0.0001$ & 16.28 & 8.35 \\
\hline Strain $\times$ Stage & 1.89 & 4,24 & $<0.0001$ & 11.09 & -11.57 \\
\hline \multicolumn{6}{|c|}{ (B) } \\
\hline Strain & 0.99 & 2,39 & $<0.0001$ & 47.84 & -7.90 \\
\hline Sex & 1.00 & 2,39 & $<0.0001$ & 49.46 & -4.80 \\
\hline Age & 1.98 & 8,80 & $<0.0001$ & 49.48 & -3.43 \\
\hline Strain $\times$ Sex & 1.00 & 2,39 & $<0.0001$ & 48.66 & -1.11 \\
\hline Strain $\times$ Age & 1.93 & 8,80 & $<0.0001$ & 46.59 & -9.05 \\
\hline Sex $\times$ Age & 1.99 & 8,80 & $<0.0001$ & 49.49 & -4.55 \\
\hline Strain $\times$ Sex $x$ Age & 1.98 & 8,80 & $<0.0001$ & 49.52 & -4.06 \\
\hline
\end{tabular}
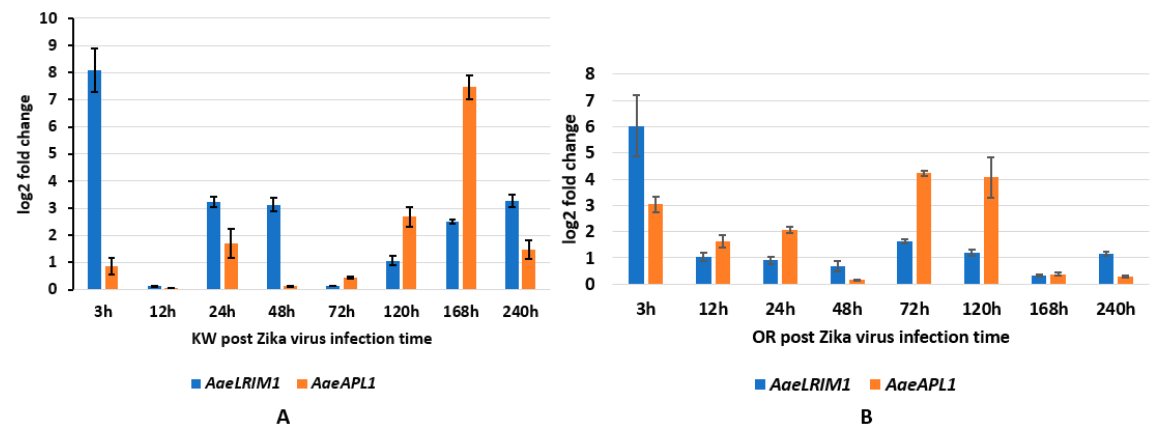

Figure 2. AaeLRIM1 and AaeAPL1 relative expression level fold changes in Aedes aegypti female infected with ZIKV. The fold change was calculated using the $2^{[- \text {average } \triangle \Delta C T]}$ method. $\Delta \mathrm{Ct}(\mathrm{Control})=\mathrm{Ct}$ (AaeLRIM1/AaeAPL1) - Ct (AeaActin); $\triangle \mathrm{Ct}$ (infected-ZIKV) = Ct (AaeLRIM1/AaeAPL1) - Ct (AeaActin); $\Delta \Delta \mathrm{Ct}=\Delta \mathrm{Ct}$ (infected-ZIKV) $-\Delta \mathrm{Ct}$ (Control). The 3, 12, 24, 48, 72, 120, 168, and $240 \mathrm{~h}$ represented gene expression post infection with ZIKV. (A) Key West strain female Ae. aegypti; (B) Orlando strain female Ae. aegypti.

\subsubsection{AaeLRIM1 and AaeAPL1 RNA Profile in Adults (Male and Female) of Ae. aegypti}

MANOVA showed significant main effects of mosquito strain, sex, and age, as well as the two-way interaction and three-way interaction (strain $\times$ sex $\times$ age). Standardized canonical coefficients showed that AaeLRIM1 contributed much more to all significant effects than AaeAPL1 (Table 2B). Because the three-way interaction was significant, we focused on pairwise comparisons of treatment groups for this effect. Specifically, we compared mosquito strains of a given sex and age (e.g., Key West, female, 1-day old vs. Orlando, female, 1-day old). For expression of AaeLRIM1, Key West was higher for 1-day, 3-day, and 10-day old mosquitoes than Orlando female Ae. aegypti. In contrast, AaeLRIM1 was higher for 5-day and 7-day Orlando than Key West female Ae. aegypti. Gene expression for AaeLRIM1 was higher for all ages of Key West male Ae. aegypti except for 1-day old males. Lowest rates of expression were observed for 1-day old male Ae. aegypti for both strains and highest rates were observed for intermediate aged mosquitoes. 
For gene expression of AaeAPL1, Orlando was higher for all ages than Key West female Ae. aegypti. Rates of expression were highest for 7-day old female mosquitoes and expression was lower among older females (10-day old) for both mosquito strains. For gene expression of AaeAPL1, Orlando was higher for all ages than Key West male Ae. aegypti, except 5-day old males. Rates of expression were highest for 5-day old and 3-day old male mosquitoes for Key West and Orlando Ae. aegypti, respectively. Gene expression was low for young and old male mosquitoes (Figure 1).

\subsection{Infection in Ae. aegypti Exposed to CHIKV and ZIKV}

In Florida, and other geographic regions of the world, there is a heterogeneity in the susceptibility of Ae. aegypti to insecticides, in part, attributable to the evolution of insecticide resistance. In particular, pyrethroid insecticides, such as permethrin, continue to be the primary means to control Ae. aegypti [39]. For this study, we deliberately included both susceptible and permethrin resistant strains of Ae. aegypti to allow for the possibility to observe differences in gene expression between the strains following ingestion of arbovirus infected blood. The susceptible strain was a long-standing laboratory colony of Ae. aegypti originating from Orlando, Florida $\left(28.53^{\circ} \mathrm{N}, 81.37^{\circ} \mathrm{W}\right)$. The resistant strain was created based on a collection of Ae. aegypti from Key West, Florida $\left(24.55^{\circ} \mathrm{N}, 81.78^{\circ} \mathrm{W}\right)$. We first tested Ae. aegypti from Key West for permethrin resistance following the use of World Health Organization (WHO) bottle bioassays (WHO 2016). Next, we subjected this population to 15 generations of permethrin selection and re-tested for permethrin resistance (resistant strain). Aedes aegypti females aged 4-days as adults were allowed to feed on blood consisting of either $6.4 \log 10 \mathrm{pfu} / \mathrm{mL}$ of ZIKV (Table 3A) or $8.0 \pm 0.09$ and $8.3 \pm 0.08 \log 10 \mathrm{pfu} / \mathrm{mL}$ of CHIKV for each strain (Table 3B). The amount of virus imbibed by mosquitoes and the viral titer at 3,7 , and 10, days post infections are described in Table 3A, B. After 10-days post infection, but not at any earlier times, we detected 100-fold higher ZIKV titer in the permethrin resistant strain than the susceptible strain $(t 4=8.12, p=0.001)$ [34]. For the CHIKV infection study, we observed no significant differences in the viral titer between the susceptible and resistant strains of Ae. aegypti (all $p>0.05)$.

Table 3. (A) Zika virus titers $(\log 10 \mathrm{pfu} / \mathrm{mL})$ in infectious blood meals and mosquitoes for Key West and Orlando strains of Aedes aegypti. (B) Chikungunya virus titers $(\log 10 \mathrm{pfu} / \mathrm{mL})$ in infectious blood meals and mosquitoes for Key West and Orlando strains of Aedes aegypti.

\begin{tabular}{cccccc}
\hline Strains & $\begin{array}{c}\text { Initial Dose in } \\
\text { Bloodmeal }\end{array}$ & Freshly Fed (3 h) & $\begin{array}{c}\text { 3 Days (72 h) } \\
\text { Post Infection }\end{array}$ & $\begin{array}{c}\text { 7 Days (168 h) } \\
\text { Post Infection }\end{array}$ & $\begin{array}{c}\text { 10 Days (240 h) } \\
\text { Post Infection }\end{array}$ \\
\hline \multicolumn{5}{c}{ (A) } \\
\hline $\begin{array}{c}\text { Key West } \\
\text { Orlando }\end{array}$ & $6.4 \pm 0.09$ & $4.30 \pm 0.0$ & $3.96 \pm 0.26$ & $4.11 \pm 1.78$ & $6.57 \pm 0.05$ \\
\hline \multicolumn{7}{c}{$(\mathbf{B})$} & $3.66 \pm 0.27$ & $3.58 \pm 1.23$ & $4.49 \pm 0.34$ \\
\hline Key West & $8.0 \pm 0.08$ & $4.17 \pm 0.39$ & $4.7 \pm 0.81$ & $5.4 \pm 0.46$ & $5.20 \pm 0.49$ \\
Orlando & $8.3 \pm 0.08$ & $4.56 \pm 0.10$ & $5.04 \pm 0.71$ & $5.81 \pm 0.48$ & $5.53 \pm 0.33$ \\
\hline
\end{tabular}

Zika virus (strain PRVABC59, GenBank accession \# KU501215.1) isolated from a human infected in Puerto Rico in 2015. Primers: ZIKV-FWD: 5'-CTTCTTATCCACAGCCGTCTC-3'; ZIKV-REV: 5'-CCAGGCTTCAACGTCGTTAT-3' . CHIKV (LaReunion strain LR2006-OPY1, GenBank KT449801) from a human infected on La Réunion Island in 2006 (Parola et al. 2006). Primers: CHIKV-FWD: 5'GTACGGAAGGTAAACTGGTATGG-3'; CHIKV-REV: 5'-TCCACCTCCCACTCCTTAAT-3'.

\subsection{AaeLRIM1 and AaeAPL1 Transcriptional Induction of ZIKV Infections in Orally Infected Ae. aegypti Females}

To characterize AaeLRIM1 and AeaAPL1 expression in response to ZIKV exposure, we measured AaeLRIM1 and AeaAPL1 expressions in orally infected Ae aegypti. MANOVA showed the significant effects of strain of Ae. aegypti, time, and their interaction (Table 4A). For the strain effect, SCCs showed that AaeLRIM1 contributed more to the significant effect than AaeAPL1 (Table 4A). Gene expression of AaeLRIM1 was significantly higher for Key West than Orlando strains. Similar contributions of gene 
expression of AaeAPL1 were observed for Orlando and Key West Ae. aegypti. For the significant time effect, SCCs showed that AaeLRIM1 contributed approximately 8-fold higher than AaeAPL1 (Table 4A). The highest gene expression of AaeLRIM1 was 3-hours post infection with the later time points having lower levels. For AaeAPL1, the highest levels were observed for 72, 120, and 168-hours post infection with other points being lower (Figure 2).

For the interaction, SCCs showed that AaeAPL1 contributed approximately 2-fold greater than AaeLRIM1 (Table 4A). We made less than all possible comparisons by comparing between strains and holding time constant (e.g., Key West, 3-hours vs. Orlando, 3-hours). Gene expression for AaeLRIM1 was highest for 3-hours post infection compared to other time points for both Key West and Orlando Ae. aegypti. All pairwise contrasts of treatment groups were significantly different from one another, except for $120 \mathrm{~h}$ post infection (Figure 2A,B).

For the significant interaction, gene expression for AaeAPL1 was highest for Key West at 168-hours post infection and for Orlando at 72- and 120-hours post infection for Ae. aegypti. All pairwise comparisons were significantly different between each other except for 24- and 48-hours post infection between the two mosquito strains (Figure 2A,B). For comparison, the amount of ZIKV imbibed by mosquitoes (3-hours) and the viral titer at 72-hours, 168-hours, and 240-hours days post infection are described in Table 3A.

To determine paralogues of AeaAPL1 expression in response to ZIKV exposure, we measured AeaLRIM3, AeaLRIM4, AeaLRIM15, AeaLRIM16, and AeaLRIM17 expressions in Ae. aegypti. MANOVA showed significant effects of the strain of Ae. aegypti, time, and their interaction (Table 4B). For the strain effect, gene expression of all five paralogues of AeaAPL1 were higher for Key West than Orlando strains, with AeaLRIM16 being the largest contributor. For the time effect, AeaLRIM17 made the largest contribution with the highest expression at $3 \mathrm{~h}$ post infection. Gene expression for the remaining paralogues were highest between 3- and 48-hours post infections (Figure 3A,B).
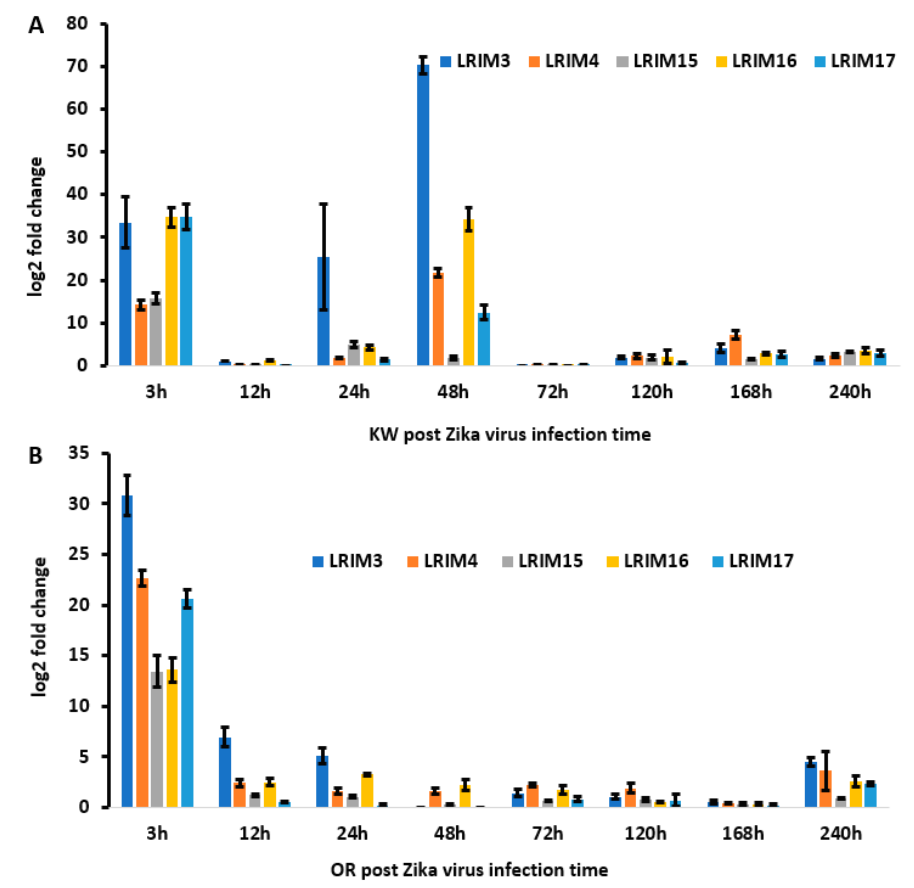

Figure 3. AaeLRIM3, AaeLRIM4, AaeLRIM15, AaeLRIM16, and AaeLRIM17 relative expression level fold changes in Aedes aegypti female infected with ZIKV. The fold change was calculated using the 2 [-average $\Delta \triangle C T]$ method. $\Delta \mathrm{Ct}(\mathrm{Control})=\mathrm{Ct}($ AaeLRIM1/AaeAPL1) - Ct (AeaActin); $\triangle \mathrm{Ct}$ (infected-ZIKV) $=\mathrm{Ct}($ AaeLRIM1/AaeAPL1) $-\mathrm{Ct}$ (AeaActin); $\Delta \Delta \mathrm{Ct}=\Delta \mathrm{Ct}$ (infected-ZIKV) $-\Delta \mathrm{Ct}$ (Control). The 3, 12, 24, $48,72,120,168$, and $240 \mathrm{~h}$ represented gene expression post infected with ZIKV. (A) KW strain female Ae. aegypti; (B) Orlando strain female Ae. aegypti. 
Table 4. (A) MANOVA results for strain and time effects of the AaeLRIM1 and AaeAPL1 RNA profile in response to Zika virus infection of Ae. aegypti. (B) MANOVA results for strain and time effects of paralogues of the AaeAPL1 RNA profile in response to Zika virus infection of Ae. aegypti.

\begin{tabular}{|c|c|c|c|c|c|c|c|c|c|c|c|c|c|c|c|c|}
\hline \multirow[t]{2}{*}{ (A) } & \multirow[t]{2}{*}{ Treatment } & \multirow[t]{2}{*}{$\begin{array}{l}\text { Pillai's } \\
\text { Trace }\end{array}$} & \multirow[t]{2}{*}{$\begin{array}{l}\text { df (Numerator and } \\
\text { Denominator }\end{array}$} & \multirow[t]{2}{*}{$p$-value } & \multicolumn{2}{|c|}{$\begin{array}{l}\text { Standardized } \\
\text { Canonical } \\
\text { Coefficients }\end{array}$} & \multirow[t]{2}{*}{ (B) } & \multirow[t]{2}{*}{ Treatment } & \multirow[t]{2}{*}{$\begin{array}{l}\text { Pillai's } \\
\text { Trace }\end{array}$} & \multirow[t]{2}{*}{$\begin{array}{l}\text { df (Num., } \\
\text { Denom.) }\end{array}$} & \multirow[t]{2}{*}{$p$-value } & \multicolumn{5}{|c|}{ Standardized Canonical Coefficients } \\
\hline & & & & & AaeLR & IA & & & & & & AaeLR1M3 & AaeLRIM4 & AaeLRIM15 & AaeLRIM16 & AaeLRIM17 \\
\hline & Strain & 0.86 & 2,31 & $<0.0001$ & 8.06 & -1.16 & & Strain & 0.96 & 5,28 & $<0.0001$ & 2.78 & 0.14 & -4.04 & 11.14 & 2.86 \\
\hline & Time & 1.94 & 14,64 & $<0.0001$ & 8.07 & -1.01 & & Time & 3.19 & 35,160 & $<0.0001$ & -0.05 & 5.94 & 4.41 & -1.81 & 8.07 \\
\hline & Strain $\times$ Time & 1.86 & 14,64 & $<0.0001$ & 2.45 & 5.84 & & Strain $\times$ Time & 3.50 & 35,160 & $<0.0001$ & 2.92 & 2.25 & -6.76 & 11.38 & 1.44 \\
\hline
\end{tabular}


For the interaction, SCCs showed that AeaLRIM16 contributed approximately 2- to 8-fold greater than the other paralogues. As previously described, we made comparisons between strains and held time constant (e.g., Key West, 3-hours vs. Orlando, 3-hours). Gene expression for AeaLRIM16 was significantly higher at 3- and 48-hour post infection for Key West than Orlando strains of Ae. aegypti. No other comparisons were significantly different from one another after adjusting the experimentwise alpha for multiple comparisons.

\subsection{AaeLRIM1 and AaeAPL1 Transcriptional Induction of CHIKV Infections in Orally Infected Ae. aegypti Females}

To characterize AaeLRIM1 and AaeAPL1 expression in response to CHIKV exposure, we measured AaeLRIM1 and AaeAPL1 expressions in orally infected Ae aegypti. MANOVA showed the significant effects of the strain of $A$ e. aegypti, time, and their interaction. For all significant treatment effects, SCCs showed that AaeLRIM1 contributed approximately 2-6-fold greater than AaeAPL1 (Table 5A). For the strain effect, gene expression of AaeLRIM1 and AaeAPL1 was significantly higher for Key West than Orlando strains. For the time effect, gene expression of AaeLRIM1 was highest at 24-hours post infection. All time points were significantly different from one another, except 3-hours versus 120-hours post infection (Figure 4). Similarly, gene expression of AaeAPL1 was highest at 72-hours post infection. All time points were significantly different from one another except the following: 3-hours versus 168 -hours, 24 -hours versus 240 -hours, and 48-hours versus 120-hours (Figure 4).
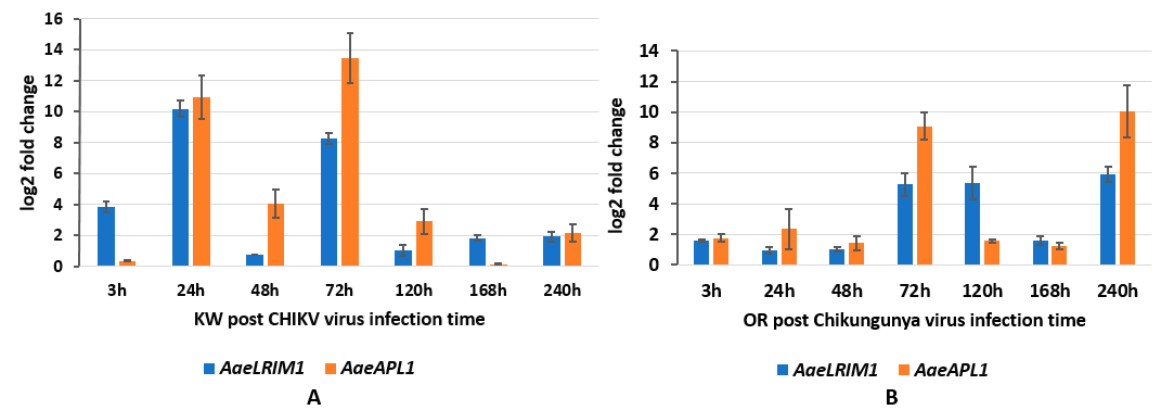

Figure 4. AaeLRIM1 and AaeAPL1 relative expression level fold changes in Aedes aegypti female infected with CHIKV. The fold change was calculated using the $2^{[- \text {average } \Delta \Delta C T]}$ method. $\Delta \mathrm{Ct}(\mathrm{Control})=$ $\mathrm{Ct}$ (AaeLRIM1/AaeAPL1) - Ct (AeaActin); $\triangle \mathrm{Ct}($ infected-CHIKV) $=\mathrm{Ct}($ AaeLRIM1/AaeAPL1) $-\mathrm{Ct}$ (AeaActin); $\Delta \Delta \mathrm{Ct}=\Delta \mathrm{Ct}$ (infected-CHIKV) $-\Delta \mathrm{Ct}$ (Control). The 3, 24, 48, 72, 120, 168, and $240 \mathrm{~h}$ represented gene expression post infected with CHIKV. (A) Key West strain female Ae. aegypti; (B) Orlando strain female Ae. aegypti.

For the significant interaction, gene expression of AaeLRIM1 was highest for 24-hours and 240-hours post infection for Key West and Orlando Ae. aegypti, respectively (Figure 4). All pairwise comparisons of treatment groups were significantly different from one another except for 48-hours and 168-hours post infection (Figure 4). The timing of the highest gene expression of AaeAPL1 was slightly different to observations of AaeLRIM1, with the highest levels observed for 72-hours and 240-hours post infection for Key West and Orlando Ae. aegypti, respectively (Figure 4). All pairwise comparisons of treatment groups were significantly different from one another except for 3-hours, 120-hours, and 168-hours post infection (Figure 4). For comparison, the amount of CHIKV imbibed by mosquitoes (3-hours) and the viral titer at 72-hours, 168-hours, and 240-hours days post infection are described in Table 3B. 
Table 5. (A) MANOVA results for strain and time effects of AaeLRIM1 and AaeAPL1 RNA profile in response to chikungunya virus infection of Ae. aegypti. (B) MANOVA results for strain and time effects of paralogues of AaeAPL1 RNA profile in response to chikungunya virus infection of Ae. aegypti.

\begin{tabular}{|c|c|c|c|c|c|c|c|c|c|c|c|c|c|c|c|c|}
\hline \multirow[t]{3}{*}{ (A) } & \multirow[t]{2}{*}{ Treatment } & \multirow[t]{2}{*}{$\begin{array}{l}\text { Pillai's } \\
\text { Trace }\end{array}$} & \multirow[t]{2}{*}{$\begin{array}{l}\text { df (Numerator and } \\
\text { Denominator) }\end{array}$} & \multirow[t]{2}{*}{$p$-value } & \multicolumn{2}{|c|}{$\begin{array}{l}\text { Standardized } \\
\text { Canonical } \\
\text { Coefficients }\end{array}$} & \multirow[t]{2}{*}{ (B) } & \multirow[t]{2}{*}{ Treatment } & \multirow[t]{2}{*}{$\begin{array}{l}\text { Pillai's } \\
\text { Trace }\end{array}$} & \multirow{2}{*}{$\begin{array}{c}\text { df (Numerator } \\
\text { and } \\
\text { Denominator) }\end{array}$} & \multirow[t]{2}{*}{$p$-value } & \multicolumn{5}{|c|}{ Standardized Canonical Coefficients } \\
\hline & & & & & AaeLR & AaeAl & & & & & & AaeLR1M3 & AaeLRIM4 & AaeLRIM15 & AaeLRIM16 & AaeLRIM17 \\
\hline & Strain & 0.60 & 2,27 & $<0.0001$ & 5.90 & 1.23 & & Strain & 0.99 & 5,24 & $<0.0001$ & 25.13 & -0.51 & -0.28 & 10.46 & -0.58 \\
\hline & Time & 1.77 & 12,56 & $<0.0001$ & 4.59 & 2.61 & & Time & 2.70 & 30,140 & $<0.0001$ & 25.50 & -0.99 & 0.22 & 11.01 & -1.32 \\
\hline & Strain $\times$ Time & 1.79 & 12,56 & $<0.0001$ & 6.20 & 0.79 & & Strain $\times$ Time & 2.31 & 30,140 & $<0.0001$ & 24.57 & -0.32 & 0.46 & 10.82 & -1.08 \\
\hline
\end{tabular}


To determine paralogues of AeaAPL1 expression in response to CHIKV exposure, we measured AeaLRIM3, AeaLRIM4, AeaLRIM15, AeaLRIM16, and AeaLRIM17 expressions in Ae. aegypti. Similar to responses to ZIKV exposure, MANOVA showed significant effects of strain of Ae. aegypti, time, and their interaction (Table 5B). In all cases, gene expression of all five paralogues of AeaAPL1 were higher for Key West than Orlando strains with AeaLRIM3 and AeaLRIM16 contributing far more than the other paralogues. For the time effect, AeaLRIM3 and AeaLRIM16 contributed far more than the other paralogues, with by far the highest expressions at 24-hours post infection. Gene expression for the remaining paralogues were similarly highest at 24-hours post infection. Gene expression for all the paralogues tended to be lowest later during the infection process (168- and 240-hour post infection).

For the interaction, SCCs showed that AeaLRIM3 contributed 2- to 76-fold greater than the other paralogues. As previously described, we made comparisons between strains and held time constant. Gene expression for AeaLRIM3 was significantly higher at 3-, 24-, and 72-hours post infection for Key West than Orlando strains of Ae. aegypti. In contrast, gene expression for AeaLRIM3 was significantly higher at 120-hours post infection for Orlando than Key West strains of Ae. aegypti (Figure 5A,B). No other comparisons were significantly different from one another.

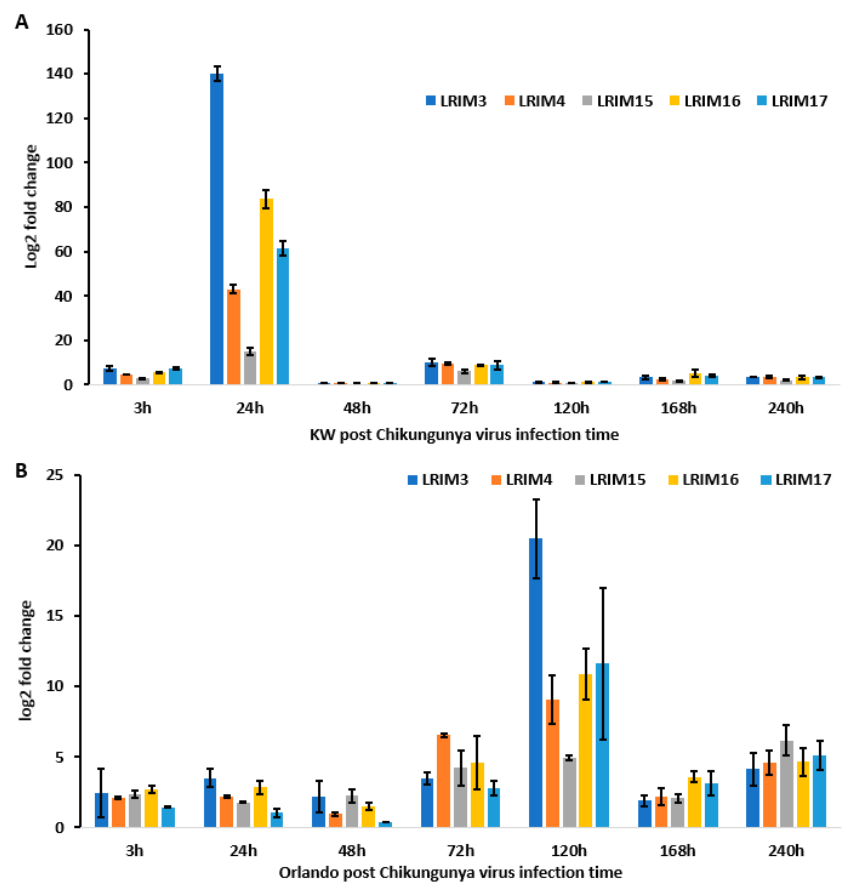

Figure 5. AaeLRIM3, AaeLRIM4, AaeLRIM15, AaeLRIM16, and AaeLRIM17 relative expression level fold changes in Aedes aegypti female infected with CHIKV. The fold change was calculated using the 2 [-average $\Delta \Delta C T]$ method. $\Delta \mathrm{Ct}(\mathrm{Control})=\mathrm{Ct}($ AaeLRIM1 $/$ AaeAPL1) $-\mathrm{Ct}($ AeaActin $) ; \Delta \mathrm{Ct}$ (infected-CHIKV) $=\mathrm{Ct}($ AaeLRIM1 $/$ AaeAPL1) $-\mathrm{Ct}($ AeaActin $) ; \Delta \Delta \mathrm{Ct}=\Delta \mathrm{Ct}$ (infected-CHIKV) $-\Delta \mathrm{Ct}(\mathrm{Control})$. The 3, $24,48,72,120,168$, and $240 \mathrm{~h}$ represented gene expression post infection with CHIKV. (A) KW strain female Ae. aegypti; (B) Orlando strain female Ae. aegypti.

\section{Discussion}

Identification and characterization of genes related to LRIM1 and APL1C revealed novel innate immune factors and furthered our understanding of their presumed molecular functions. Waterhouse et al. (2010) used comparative sequenced genomes: An. gambiae, Ae. aegypti, and Cx. quinquefasciatus revealed that mosquito LRIM proteins can be classified into four distinct subfamilies by a variable number of LRRs [16]. Our phylogenetic tree of paralogue showed that AaeAPL1 (or AaeLRIM2) sequence-structure-function was most closely related to AaeLRIM4.

We analyzed developmental changes in the gene expression of LRR-containing proteins in Ae. aegypti eggs, larvae, pupae, and adults. The nucleus gene LRR-containing proteins, AaeLRIM1, 
AaeAPL1, and the other five paralogues are expressed $<100$-fold lower than the other nuclear genes, such as defensin, during all developmental stages examined [40]. Our data show that the expression of AaeLRIM1 (AAEL012086), AaeAPL1 (AAEL009520-RA), and the other five paralogues is not only regulated by development, but also by the varying environmental origin (or permethrin resistant selected strain) of mosquito strains in Ae. aegypti. For both immature and adult stages, we observed higher expression of AaeLRIM1 than AaeAPL1 in Key West Ae. aegypti and higher expression of AaeAPL1 than AaeLRIM1 in Orlando Ae. aegypti. These differences in responses may be attributable to differences in insecticide resistance among the Orlando (permethrin susceptible) and Key West (permethrin resistant) strains of Ae. aegypti. However, we are unable to rule out that other differences between these two strains of Ae. aegypti (e.g., geographic origin and founder effects) may contribute to the observed differences in gene expressions. For the immature, but not adult stages, expression of AaeAPL1 and AaeLRIM1 increased with the developmental stage. The strain effect for the analyses were modified by interactions with other factors, suggesting complex interactions between gene expression and immature stage, adult age, and sex. To our knowledge, this is the first exploration of AaeLRIM gene expression during Ae. aegypti development.

In Anopheles, two leucine-rich repeat (LRR) protein related genes, LRIM1 and APL1, have been shown to strongly affect $P$. berghei development in the mosquito midgut and have been identified as major mosquito factors that regulate parasite loads [11,13-15,41-44]. In Ae. aegypti, the likely LRIM1 orthologue is upregulated with other immune genes following infection with Wolbachia bacteria, resulting in immune activation and shortened mosquito life spans [45]. However, there is no information available for revealing LRIM1 and APL1 affected by arboviruses. Our data demonstrates that gene expression of LRR-containing proteins, AaeLRIM1 and AaeAPL1, not only affect regulated parasites in the Anopheles, but are also altered by arbovirus infection in Ae. aegypti. Biophysical analysis of An. gambiae LRR proteins, APL1A1, APL1B, and APL1C, can arrange an extended, flexible heterodimer with LRIM1, providing a repertoire of functional innate immune complexes to protect An. gambiae from a diverse array of pathogens [14]. Future studies are needed to identify how proteins, AaeLRIM1 and AaeAPL1, may influence progression of infection of arboviruses in Ae. aegypti. Some of the highest changes in expression of AaeAPL1 and AaeLRIM1 for both strains of Ae. aegypti occurred 24-72 $\mathrm{h}$ post infection with CHIKV, which approximates the time when Ae. aegypti acquire disseminated infections [24]. However, this response appears to be earlier for the Key West strain (approximately $24 \mathrm{~h}$ ), which may suggest alterations in immune responses between permethrin susceptible and resistant Ae. aegypti (Shin et al. unpublished data). Changes in the expression of AaeLRIM1 and AaeAPL1 followed a different pattern for ZIKV infected mosquitoes, suggesting gene expression changes depend on the particular arbovirus. For both strains of Ae. aegypti, AaeLRIM1 tended to be highest early during infection and declined at later points. In contrast, expression of AaeAPL1 was low early during infection and higher at later measured times.

We also described the gene expression of five paralogues of AaeAPL1 (AaeLRIM3, AaeLRIM4, AaeLRIM15, AaeLRIM16, and AaeLRIM17) in Ae. aegypti following ingestion of arbovirus infected blood. The relative contributions of the five paralogues of AaeAPL1 differed in response to infection with either ZIKV or CHIKV, which may suggest differences in mounting an immune response between viruses of different families. Gene expression of AeaLRIM16 and AeaLRIM17 made the largest contributions to significant treatment effects for mosquitoes orally exposed to ZIKV. Along the same lines, AeaLRIM3 and AeaLRIM16 made the largest contributions to significant treatment effects for mosquitoes following oral ingestion of CHIKV infected blood. However, there were some common features observed in the gene expression of the paralogues of AaeAPL1. Gene expression of all five paralogues of AaeAPL1 were usually the highest within the first 48-hours following ingestion of ZIKV or CHIKV infected blood, with lower rates of expression later during the infection process.

Studying the immune system of mosquitoes provides insights into significant opportunities to bridge tissue damage, immune invasions mechanisms, and the immune response against pathogens [4]. More importantly, unveiling a newfound understanding of mosquito immunity will shed light on 
the fight against disease-spreading pathogens, including ZIKV and CHIKV. Understanding the mechanisms that allow pathogens to grow and replicate in mosquitoes will provide insights into the mechanisms of mosquito-pathogen interactions. Finding exact immune evasion strategies of pathogens will help produce novel strategies that are effective at controlling them.

\section{Materials and Methods}

\subsection{Mosquito Strains and Developmental Stages of Aedes aegypti}

Ae. aegypti larvae were collected from Key West $\left(24.55^{\circ} \mathrm{N}, 81.78^{\circ} \mathrm{W}\right)$, Florida, USA since 2011 and were initially tested for permethrin resistance, then subjected to permethrin selection for 15 generations and again assayed for resistance strain [34]. Key West strain Ae. aegypti, referred to as the resistant strain, was maintained at the Florida Medical Entomology Laboratory (FMEL) in Vero Beach, FL, USA. The Orlando population of Ae. aegypti was collected from Orlando, FL, USA and reared in the Mosquito and Fly Research Unit, Center for Medical, Agricultural and Veterinary Entomology, ARS-USDA in Gainesville, FL since 1952. The Orlando strain is recognized as a permethrin susceptible strain of Ae. aegypti [46]. For the experiments, mosquito eggs were hatched and reared in a rearing chamber at $27^{\circ} \mathrm{C}$ [40]. We sampled eggs, larvae, pupae, and adults to measure developmentally regulated gene expression of Leucine-Rich Repeat Proteins in Ae. aegypti. We collected $100 \mu \mathrm{g}$ of eggs, 20 larvae at the third instar stage, 20 pupae, 10 adult mosquitoes, 10 males and 10 females at 1 -day-old (teneral), 3-day-old, 5-day-old, 7-day-old, and 10-day-old adults. The experiments were repeated three times.

\subsection{Chikungunya Virus and Zika Virus Infection}

Four-day-old female adults were fed blood containing either CHIKV, ZIKV, or blood without virus as the control $[34,40]$. Isolates of the Indian Ocean lineage of CHIKV (LR2006-OPY1, GenBank accession: KT449801) from Réunion and the Asian lineage of ZIKV (strain PRVABC59, GenBank accession \# KU501215.1) from Puerto Rico were cultured in African green monkey (Vero) cells and used in the mosquito infection study. Ten mosquitos were used in each sample and three independent experiments were conducted for each time point. The detailed procedures were described in the previous publication $[34,40]$.

Arboviruses were propagated on monolayers of African green monkey kidney (Vero) cells at a multiplicity of infection of 0.1 and environmental conditions of $37{ }^{\circ} \mathrm{C}$ and $5 \% \mathrm{CO}_{2}$. Monolayers of cells were covered with $24 \mathrm{~mL}$ media (M199 medium supplemented with $10 \%$ fetal bovine serum, penicillin/streptomycin, and mycostatin). For CHIKV and ZIKV, we collected the media with virus on days three and six, respectively, and mixed it with defibrinated bovine blood containing $0.005 \mathrm{M}$ ATP. Mosquitoes were allowed to feed on infected blood for one hour feeding trials using a Hemotek membrane feeding system. Samples of blood were taken during feeding trials to allow for quantification of the amount of virus presented to mosquitoes. We also allowed mosquitoes to feed on a blood and media mixture without the presence of arboviruses (controls). Mosquitoes were fed 8.0-8.3 $\log 10 \mathrm{pfu} / \mathrm{mL}$ of CHIKV for each strain and $6.4 \log 10 \mathrm{pfu} / \mathrm{mL}$ of ZIKV.

Following feeding trials, fully engorged mosquitoes were sorted using light microscopy $(10 \times)$ (Carson, COPYRIGHT 2006 USA, UK, HK, CHINA, Made in China) and held in cages (h by d: $10 \mathrm{~cm}$ by $10 \mathrm{~cm}$ ) maintained at a 12:12 h light:dark photoperiod and $30^{\circ} \mathrm{C}$. Mosquitoes were provided with an oviposition substrate and $10 \%$ sucrose solution on cotton pads. Cohorts of mosquitoes were killed and stored at $-80^{\circ} \mathrm{C}$ at the following sample periods after ingesting infected blood: 3-h, 12-h (ZIKV only), 24-hours, 48-hours, 72-hours, 120-hours, 168-hours, and 240-hours. Mosquitoes were deprived of sucrose, but not water 1-day before trials used to measure transmission on 3, 5, 7, and 10 days following ingestion of ZIKV and CHIKV infection blood. 


\subsection{RNA Extraction}

Samples were homogenized with a plastic pestle in $1.5 \mathrm{~mL}$ tubes. Total RNAs were extracted using TRIzol reagent according to the manufacturer's instructions (Ambion, Life Technologies, Carlsbad, CA, USA) following the standard protocol [40]. The RNA samples were digested by DNase I (RNase-free), according to the manufacturer's instructions (Thermo Scientific, Wilmington, DE, USA). The purified RNA samples were quantitated by an NANODROP 2000 Spectrophotometer (Thermo Scientific, Wilmington, DE, USA).

\section{4. cDNA Synthesis and qPCR Amplification}

cDNAs from $2 \mu \mathrm{g}$ of total purified RNA were synthesized using a Cloned AMV First-Strand cDNA Synthesis Kit Invitrogen ${ }^{\mathrm{TM}}$ and Oligo (dT) 20 primer, according to the manufacturer's instructions (Invitrogen, Carlsbad, CA). The reaction was terminated by heat inactivation at $95^{\circ} \mathrm{C}$ for $5 \mathrm{~min}$. The cDNA samples for qPCR from the developmental stages, infected treatment, and controls were diluted by adding $80 \mu \mathrm{L}$ dd $_{2} \mathrm{O}$ to $20 \mu$ reaction solution [47].

The quantitative PCR (qPCR) assay for target genes, AaeLRIM1 and AaeAPL1, and reference gene, AaeActin, in Ae. aegypti was achieved using a BIO-RAD C1000 Touch Thermal Cycler, CFX $96^{\mathrm{TM}}$ Real-Time System (BIO-RAD, Hercules, CA, USA). The qPCR reaction mixture with a volume of $15 \mu \mathrm{l}$ in Multiwell Plates 96 contained $1 \mu \mathrm{l}$ diluted cDNA, $0.5 \mu \mathrm{M}$ primers, and $1 \times$ master mix of PowerUP SYBR ${ }^{\circledR}$ Green Master Mix (Applied Biosystems, Thermo Fisher Scientific, Foster City, CA, USA). In every qPCR run, AaeActin was employed as an internal control to normalize for variation in the amount of cDNA template. The PCR primers for AaeLRPIM, and AaeAPL1 genes were designed from the coding region based on GenBank, Accession Number using Primer3 http:/ / primer3.ut.ee (accessed on: Jul 20, 2017) (Table 6). The qPCR thermal cycling parameters were the same as previous publication [48]. Relative expression levels were calculated as follows for the developmental stages. First, AaeLRIM/AaeAPL1 transcript levels relative to a standard (AaeActin) were calculated using the formula, $\triangle \mathrm{CT}=\mathrm{CT}$ (AaeLRIM/AaeAPL1) - CT (AaeActin). Second, an average $\triangle \mathrm{CT}$ value for each sample was calculated. Third, relative expression levels were calculated using the equation, $10,000 \times 2^{[- \text {average } \Delta \mathrm{CT}]}[40,48]$. Relative expression levels were calculated as follows for the treatment and control adults. First, AaeLRIM1 or AaeAPL1 transcript levels relative to a standard (AaeActin) were calculated using the formula, $\triangle \mathrm{CT}=\mathrm{CT}$ (AaeLRIM1/AaeAPL1) $-\mathrm{CT}$ (AaeActin). Then, $\triangle \triangle \mathrm{CT}=\Delta \mathrm{CT}$ (infected) $-\triangle \mathrm{CT}$ (control) value for each sample was calculated. Third, relative expression levels were calculated using the equation, $1 \times 2^{[- \text {average } \Delta \Delta C \mathrm{CT}]}[40,48-51]$.

Table 6. Primers from Aedes aegypti for qPCR reaction.

\begin{tabular}{|c|c|c|c|c|}
\hline Gene ID & Accession & Gene Name & Primer Name & Primer Sequence $\left(5^{\prime}-3^{\prime}\right)$ \\
\hline \multirow[t]{2}{*}{ AAEL012086 } & XM_001662191.2 & AaeLRIM1 & AaeLRIM1-086-1011F & TGACAACCGGGTTAAGGAAG \\
\hline & & & AaeLRIM1-086-1198R & TGGCCAAATCATTGTTCTCA \\
\hline \multirow[t]{2}{*}{ AAEL024406 ${ }^{1}$} & XM_021843329.1 & AaeAPL1 & AaeAPL1-406-115F & TCAACCCAGCCTCCAGATAC \\
\hline & & & AaeAPL1-406-275R & TCAGCAGTTTCACCACTTGC \\
\hline \multirow[t]{2}{*}{ AAEL010132 } & XM_001660603.2 & AaeLRIM3 & AaeLRIM3-132-166F & TGTAGCCCGCAATAATCACA \\
\hline & & & AaeLRIM3-132-405R & CTGAAGTGCTCCGTTGAACA \\
\hline \multirow[t]{2}{*}{ AAEL010128 } & XM_001660601.2 & AaeLRIM4 & AaeLRIM4-128-612F & TGTAGCCCGCAATAATCACA \\
\hline & & & AaeLRIM4-128-830R & GCCAGATTAAGCTCCACGAG \\
\hline \multirow[t]{2}{*}{ AAEL007103 } & XM_001658083.2 & AaeLRIM15 & AaeLRIM15-103-1522F & ATGGTATTGCGTGGAGGAAG \\
\hline & & & AaeLRIM15-103-1676R & АTCСТАTCAACCGСССТTCT \\
\hline \multirow[t]{2}{*}{ AAEL008658 } & XM_021855914.1 & AaeLRIM16 & AaeLRIM16-658-299F & ACACCTTCGAGAAAGCGAAA \\
\hline & & & AaeLRIM16-658-541R & TCAACATGGGCAAATGAGAA \\
\hline \multirow{2}{*}{ AAEL010125 } & XM_001660604.2 & AaeLRIM17 & AaeLRIM17-125-555F & GCAGTACAATTCGCTGACCA \\
\hline & & & AaeLRIM17-125-718R & CCTTAAGCCGATTGAAGCTG \\
\hline \multirow[t]{2}{*}{ AAEL011197 } & XM_001655125.2 & AaeActin & AaeActin-197-152F & AGGACTCGTACGTCGGTGAC \\
\hline & & & AaeActin-197-590R & CGTTCAGTCAGGATCTTC \\
\hline
\end{tabular}

${ }^{1}$ AAEL024406 is the same gene as AAEL009520-RA. 


\subsection{RNA-Seq Library Sequencing, Data Mining, and RNA-seq Analysis}

RNA sequencing and RNA-seq analysis were featured in a previous publication [34]. Gene expression was assessed by counting the number of mapped reads for each transcript [52]. Significantly up- and downregulated genes were determined using the adjusted $p$-value (p-adj), log2 fold-change $(\log 2 \mathrm{FC})$, or both for downstream analysis. The RNA-seq data have been deposited to NCBI (https: / / www.ncbi.nlm.nih.gov/gds/?term=GSE118858). RNA-seq analysis data provided 60 leucine-rich repeat related transcriptions in the Ae. aegypti in response to Zika virus (Table S1A-D).

\subsection{Statistical Analysis}

For this study, we were interested in measuring the magnitude of gene expression of components of the leucine-rich repeats (LRR)-containing domain as well as the relationship between gene expressions. So, we used a multivariate analysis of variance approach (MANOVA), which enabled us to simultaneously measure various treatment effects (e.g., developmental stage, infection with ZIKV and CHIKV) on the expression of multiple genes. Further, the MANOVA is associated with standardized canonical coefficients, which describe the relative contribution of each of the variables (genes) to a treatment effect and their direction to one another (positive or negative) [53].

Multivariate analysis of variance (MANOVA) and ANOVA were used to measure developmentally regulated gene expression of AaeLRIM1 and AaeAPL1. The relative contribution and relationship of AaeLRIM1 and AaeAPL1 to developmental treatment effects were assessed using standardized canonical coefficients (SCC) (PROC GLM, SAS 9.22). When significant effects were detected, we used univariate comparisons among treatment least-squares means for the developmental stages (Tukey-Kramer method). Separate analyses were performed for the immature stages and adult stages. Similarly, we used MANOVA to measure the expression of AaeLRIM1 or AaeAPL1 following ingestion of CHIKV and ZIKV infected blood. We tested for all main treatment factors and interactions.

Supplementary Materials: Supplementary materials can be found at http:/ /www.mdpi.com/1422-0067/20/3/ 615/s1. Table S1A-D: Female Aedes aegypti transcriptomic RNA-seq data show Leucine-Rich Repeat Proteins 7-days post infection or post injection between Key west and Orlando strains. Figure S1. Evolutionary analysis of paralogues of AeaAPL1 of the Ae. Aegypti. Figure S2A-E. AaeLRIM3, AaeLRIM4, AaeLRIM15, AaeLRIM16, and AaeLRIM17 relative expression in the all developmental stages.

Author Contributions: Conceptualization, L.Z., and B.W.A.; Methodology, L.Z., and B.W.A.; Software, B.W.A. and L.Z.; Validation, L.Z., and B.W.A.; Formal Analysis, L.Z., and B.W.A.; Investigation, L.Z., B.W.A. and D.S.; Resources, L.Z., B.W.A. and D.S.; Data Curation, L.Z.; Writing-Original Draft Preparation, L.Z.; Writing一Review \& Editing, L.Z., B.W.A. and D.S.; Visualization, L.Z., and B.W.A.; Supervision, L.Z.; Project Administration, L.Z.; Funding Acquisition, L.Z. and B.W.A.

Funding: This research was funded by the Florida Department of Agriculture and Consumer Services: Contract Numbers 025364, 024246, 023557, 020190 and 021090 to Jorge Rey and Contract Number 020180 to Walter Tabachnick, Contract Numbers 021803 and 022399 to Liming Zhao and Barry Alto.

Acknowledgments: We thank Jordan Norus, Bradley Eastmond, Melissa Williams, Daniel Velez, Ayse Civana, Keenan Wiggins, and James Newman of the Florida Medical Entomology Laboratory, University of Florida for their excellent technical support. We would also like to thank Jorge Rey and Walter Tabachnick for their support. The isolate of Zika virus was graciously provided by the Centers for Disease Control and Prevention. The Indian Ocean lineage of chikungunya virus was kindly provided by Robert Tesh (World Reference Center for Emerging Viruses and Arboviruses, through the University of Texas Medical Branch in Galveston, Texas) to the UF-FMEL. We thank Mosquito and Fly Research Unit, Center for Medical, Agricultural and Veterinary Entomology, ARS-USDA for providing us with Ae. aegypti from Orlando, Florida.

Conflicts of Interest: All authors declare no conflicts of interest.

\section{Abbreviations}

$\begin{array}{ll}\text { ZIKV } & \text { Zika Virus } \\ \text { CHIKV } & \text { Chikungunya Virus } \\ \text { LRR } & \text { Leucine-Rich Repeats } \\ \text { LRIM1 } & \text { Leucine-Rich Repeat Immune Protein 1 } \\ \text { APL1 } & \text { Anopheles Plasmodium-responsive Leucine-rich Repeat } 1\end{array}$




\section{References}

1. $\mathrm{Ng}, \mathrm{A} . ;$ Xavier, R.J. Leucine-rich repeat (LRR) proteins: Integrators of pattern recognition and signaling in immunity. Autophagy 2011, 7, 1082-1084. [CrossRef] [PubMed]

2. Ausubel, F.M. Are innate immune signaling pathways in plants and animals conserved? Nat. Immunol. 2005, 6, 973-979. [CrossRef] [PubMed]

3. Medzhitov, R. Recognition of microorganisms and activation of the immune response. Nature 2007, 449, 819-826. [CrossRef] [PubMed]

4. Kumar, A.; Srivastava, P.; Sirisena, P.; Dubey, S.K.; Kumar, R.; Shrinet, J.; Sunil, S. Mosquito Innate Immunity. Insects 2018, 9, 95. [CrossRef] [PubMed]

5. Kantor, A.M.; Dong, S.; Held, N.L.; Ishimwe, E.; Passarelli, A.L.; Clem, R.J.; Franz, A.W. Identification and initial characterization of matrix metalloproteinases in the yellow fever mosquito, Aedes aegypti. Insect. Mol. Biol. 2017, 26, 113-126. [CrossRef] [PubMed]

6. Dong, S.; Kantor, A.M.; Lin, J.; Passarelli, A.L.; Clem, R.J.; Franz, A.W. Infection pattern and transmission potential of chikungunya virus in two New World laboratory-adapted Aedes aegypti strains. Sci. Rep. 2016, 6, 24729. [CrossRef] [PubMed]

7. Houk, E.J.; Hardy, J.L.; Chiles, R.E. Permeability of the midgut basal lamina in the mosquito, Culex tarsalis Coquillett (Insecta, Diptera). Acta Trop. 1981, 38, 163-171. [PubMed]

8. Passarelli, A.L. Barriers to success: How baculoviruses establish efficient systemic infections. Virology 2011, 411, 383-392. [CrossRef]

9. Bartholomay, L.C.; Michel, K. Mosquito Immunobiology: The Intersection of Vector Health and Vector Competence. Annu. Rev. Entomol. 2018, 63, 145-167. [CrossRef]

10. Bartholomay, L.C.; Cho, W.L.; Rocheleau, T.A.; Boyle, J.P.; Beck, E.T.; Fuchs, J.F.; Liss, P.; Rusch, M.; Butler, K.M.; $\mathrm{Wu}$, R.C.; et al. Description of the transcriptomes of immune response-activated hemocytes from the mosquito vectors Aedes aegypti and Armigeres subalbatus. Infect. Immunity 2004, 72, 4114-4126. [CrossRef]

11. Upton, L.M.; Povelones, M.; Christophides, G.K. Anopheles gambiae blood feeding initiates an anticipatory defense response to Plasmodium berghei. J. Innate Immunity 2015, 7, 74-86. [CrossRef] [PubMed]

12. Habtewold, T.; Povelones, M.; Blagborough, A.M.; Christophides, G.K. Transmission blocking immunity in the malaria non-vector mosquito Anopheles quadriannulatus species A. PLoS Pathog. 2008, 4, e1000070. [CrossRef] [PubMed]

13. Povelones, M.; Waterhouse, R.M.; Kafatos, F.C.; Christophides, G.K. Leucine-rich repeat protein complex activates mosquito complement in defense against Plasmodium parasites. Science 2009, 324, 258-261. [CrossRef] [PubMed]

14. Williams, M.; Summers, B.J.; Baxter, R.H. Biophysical analysis of Anopheles gambiae leucine-rich repeat proteins APL1A1, APL1B [corrected] and APL1C and their interaction with LRIM1. PLoS ONE 2015, 10, e0118911.

15. Fraiture, M.; Baxter, R.H.; Steinert, S.; Chelliah, Y.; Frolet, C.; Quispe-Tintaya, W.; Hoffmann, J.A.; Blandin, S.A.; Levashina, E.A. Two mosquito LRR proteins function as complement control factors in the TEP1-mediated killing of Plasmodium. Cell Host Microbe 2009, 5, 273-284. [CrossRef]

16. Waterhouse, R.M.; Povelones, M.; Christophides, G.K. Sequence-structure-function relations of the mosquito leucine-rich repeat immune proteins. BMC Genomics 2010, 11, 531. [CrossRef]

17. Riehle, M.M.; Xu, J.; Lazzaro, B.P.; Rottschaefer, S.M.; Coulibaly, B.; Sacko, M.; Niare, O.; Morlais, I.; Traore, S.F.; Vernick, K.D. Anopheles gambiae APL1 is a family of variable LRR proteins required for Rel1-mediated protection from the malaria parasite, Plasmodium berghei. PLoS ONE 2008, 3, e3672. [CrossRef]

18. Blandin, S.A.; Wang-Sattler, R.; Lamacchia, M.; Gagneur, J.; Lycett, G.; Ning, Y.; Levashina, E.A.; Steinmetz, L.M. Dissecting the genetic basis of resistance to malaria parasites in Anopheles gambiae. Science 2009, 326, 147-150. [CrossRef]

19. Kwon, H.; Arends, B.R.; Smith, R.C. Late-phase immune responses limiting oocyst survival are independent of TEP1 function yet display strain specific differences in Anopheles gambiae. Parasit Vectors 2017, 10, 369. [CrossRef]

20. Smith, R.C.; Barillas-Mury, C. Plasmodium Oocysts: Overlooked Targets of Mosquito Immunity. Trends Parasitol. 2016, 32, 979-990. [CrossRef] 
21. Tsetsarkin, K.A.; Chen, R.; Weaver, S.C. Interspecies transmission and chikungunya virus emergence. Curr. Opin. Virol. 2016, 16, 143-150. [CrossRef] [PubMed]

22. Lanciotti, R.S.; Valadere, A.M. Transcontinental movement of Asian genotype chikungunya virus. Emerg. Infect. Dis. 2014, 20, 1400-1402. [CrossRef] [PubMed]

23. Leparc-Goffart, I.; Nougairede, A.; Cassadou, S.; Prat, C.; de Lamballerie, X. Chikungunya in the Americas. Lancet 2014, 383, 514. [CrossRef]

24. Alto, B.W.; Wiggins, K.; Eastmond, B.; Velez, D.; Lounibos, L.P.; Lord, C.C. Transmission risk of two chikungunya lineages by invasive mosquito vectors from Florida and the Dominican Republic. PLoS Negl. Trop. Dis. 2017, 11, e0005724. [CrossRef] [PubMed]

25. Powers, A.M. How Chikungunya Virus Virology Affects Its Epidemiology and Transmission: Implications for Influencing Public Health. J. Infect. Dis. 2016, 214, S449-S452. [CrossRef] [PubMed]

26. Caglioti, C.; Lalle, E.; Castilletti, C.; Carletti, F.; Capobianchi, M.R.; Bordi, L. Chikungunya virus infection: An overview. New Microbiol. 2013, 36, 211-227. [PubMed]

27. Gasque, P.; Bandjee, M.C.; Reyes, M.M.; Viasus, D. Chikungunya Pathogenesis: From the Clinics to the Bench. J. Infect. Dis. 2016, 214, S446-S448. [CrossRef]

28. Solomon, T.; Baylis, M.; Brown, D. Zika virus and neurological disease-approaches to the unknown. Lancet Infect. Dis. 2016, 16, 402-404. [CrossRef]

29. Lanciotti, R.S.; Lambert, A.J.; Holodniy, M.; Saavedra, S.; Signor, L.e.C. Phylogeny of Zika Virus in Western Hemisphere, 2015. Emerg. Infect. Dis. 2016, 22, 933-935. [CrossRef]

30. Sacramento, C.Q.; de Melo, G.R.; de Freitas, C.S.; Rocha, N.; Hoelz, L.V.; Miranda, M.; Fintelman-Rodrigues, N.; Marttorelli, A.; Ferreira, A.C.; Barbosa-Lima, G.; et al. The clinically approved antiviral drug sofosbuvir inhibits Zika virus replication. Sci. Rep. 2017, 7, 40920. [CrossRef]

31. Cuevas, E.L.; Tong, V.T.; Rozo, N.; Valencia, D.; Pacheco, O.; Gilboa, S.M.; Mercado, M.; Renquist, C.M.; González, M.; Ailes, E.C.; et al. Preliminary Report of Microcephaly Potentially Associated with Zika Virus Infection During Pregnancy—Colombia, January-November 2016. MMWR Morb. Mortal. Wkly. Rep. 2016, 65, 1409-1413. [CrossRef] [PubMed]

32. Pinto-Díaz, C.A.; Rodríguez, Y.; Monsalve, D.M.; Acosta-Ampudia, Y.; Molano-González, N.; Anaya, J.M.; Ramírez-Santana, C. Autoimmunity in Guillain-Barré syndrome associated with Zika virus infection and beyond. Autoimmunity Rev. 2017. [CrossRef] [PubMed]

33. Dong, S.; Behura, S.K.; Franz, A.W.E. The midgut transcriptome of Aedes aegypti fed with saline or protein meals containing chikungunya virus reveals genes potentially involved in viral midgut escape. BMC Genomics 2017, 18, 382. [CrossRef] [PubMed]

34. Zhao, L.; Alto, B.W.; Shin, D.; Yu, F. The Effect of Permethrin Resistance on Aedes aegypti Transcriptome Following Ingestion of Zika Virus Infected Blood. Viruses 2018, 10, 470. [CrossRef] [PubMed]

35. Etebari, K.; Hegde, S.; Saldaña, M.A.; Widen, S.G.; Wood, T.G.; Asgari, S.; Hughes, G.L. Global Transcriptome Analysis of Aedes aegypti Mosquitoes in Response to Zika Virus Infection. mSphere 2017, 2, e00456-17. [CrossRef] [PubMed]

36. Shrinet, J.; Srivastava, P.; Sunil, S. Transcriptome analysis of Aedes aegypti in response to mono-infections and co-infections of dengue virus-2 and chikungunya virus. Biochem. Biophys. Res. Commun. 2017, 492, 617-623. [CrossRef] [PubMed]

37. Angleró-Rodríguez, Y.I.; MacLeod, H.J.; Kang, S.; Carlson, J.S.; Jupatanakul, N.; Dimopoulos, G. Molecular Responses to Zika Virus: Modulation of Infection by the Toll and Jak/Stat Immune Pathways and Virus Host Factors. Front. Microbiol. 2017, 8, 2050. [CrossRef]

38. Kumar, S.; Stecher, G.; Tamura, K. MEGA7: Molecular Evolutionary Genetics Analysis Version 7.0 for Bigger Datasets. Mol. Biol. Evol. 2016, 33, 1870-1874. [CrossRef]

39. Smith, L.B.; Kasai, S.; Scott, J.G. Pyrethroid resistance in Aedes aegypti and Aedes albopictus: Important mosquito vectors of human diseases. Pestic Biochem. Physiol. 2016, 133, 1-12. [CrossRef]

40. Zhao, L.; Alto, B.W.; Smartt, C.T.; Shin, D. Transcription Profiling for Defensins of Aedes aegypti (Diptera: Culicidae) During Development and in Response to Infection with Chikungunya and Zika Viruses. J. Med. Entomol. 2017. [CrossRef]

41. Riehle, M.M.; Markianos, K.; Niaré, O.; Xu, J.; Li, J.; Touré, A.M.; Podiougou, B.; Oduol, F.; Diawara, S.; Diallo, M.; et al. Natural malaria infection in Anopheles gambiae is regulated by a single genomic control region. Science 2006, 312, 577-579. [CrossRef] [PubMed] 
42. Osta, M.A.; Christophides, G.K.; Kafatos, F.C. Effects of mosquito genes on Plasmodium development. Science 2004, 303, 2030-2032. [CrossRef] [PubMed]

43. Osta, M.A.; Christophides, G.K.; Vlachou, D.; Kafatos, F.C. Innate immunity in the malaria vector Anopheles gambiae: Comparative and functional genomics. J. Exp. Biol. 2004, 207, 2551-2563. [CrossRef] [PubMed]

44. Blandin, S.; Shiao, S.H.; Moita, L.F.; Janse, C.J.; Waters, A.P.; Kafatos, F.C.; Levashina, E.A. Complement-like protein TEP1 is a determinant of vectorial capacity in the malaria vector Anopheles gambiae. Cell 2004, 116, 661-670. [CrossRef]

45. Kambris, Z.; Cook, P.E.; Phuc, H.K.; Sinkins, S.P. Immune activation by life-shortening Wolbachia and reduced filarial competence in mosquitoes. Science 2009, 326, 134-136. [CrossRef] [PubMed]

46. Estep, A.S.; Sanscrainte, N.D.; Waits, C.M.; Louton, J.E.; Becnel, J.J. Resistance Status and Resistance Mechanisms in a Strain of Aedes aegypti (Diptera: Culicidae) From Puerto Rico. J. Med. Entomol. 2017. [CrossRef] [PubMed]

47. Zhao, L.; Alto, B.W.; Duguma, D. Transcriptional Profile for Detoxification Enzymes AeaGGT1 and AaeGGT2 From Aedes aegypti (Diptera: Culicidae) in Response to Larvicides. J. Med. Entomol. 2017, 54, 878-887. [CrossRef]

48. Zhao, L.; Pridgeon, J.W.; Becnel, J.J.; Clark, G.G.; Linthicum, K.J. Cytochrome c gene and protein expression: Developmental regulation, environmental response, and pesticide sensitivity in Aedes aegypti. J. Med. Entomol. 2008, 45, 401-408. [CrossRef]

49. Portereiko, M.F.; Sandaklie-Nikolova, L.; Lloyd, A.; Dever, C.A.; Otsuga, D.; Drews, G.N. NUCLEAR FUSION DEFECTIVE1 encodes the Arabidopsis RPL21M protein and is required for karyogamy during female gametophyte development and fertilization. Plant Physiol. 2006, 141, 957-965. [CrossRef]

50. Livak, K.J.; Schmittgen, T.D. Analysis of relative gene expression data using real-time quantitative PCR and the 2(-Delta Delta C(T)) Method. Methods 2001, 25, 402-408. [CrossRef]

51. Portereiko, M.F.; Lloyd, A.; Steffen, J.G.; Punwani, J.A.; Otsuga, D.; Drews, G.N. AGL80 is required for central cell and endosperm development in Arabidopsis. Plant Cell 2006, 18, 1862-1872. [CrossRef] [PubMed]

52. Yao, J.Q.; Yu, F. DEB: A web interface for RNA-seq digital gene expression analysis. Bioinformation 2011, 7, 44-45. [CrossRef] [PubMed]

53. Scheiner, S.M. Multiple response variables and multispecies interactions. In Design and Analysis of Ecological Experiments; Scheiner, S.M., Gurevitch, J., Eds.; Chapman \& Hall: New York, NY, USA, 1993; pp. $94-112$. 